\title{
Paratesticular Mammary-Type Myofibroblastoma
}

National Cancer Institute

\section{Source}

National Cancer Institute. Paratesticular Mammary-Type Myofibroblastoma. NCI

Thesaurus. Code C162500.

An extramammary myofibroblastoma that arises from the paratesticular region. 